Belda, Enrique, Dignidad y discapacidad. Una perspectiva desde los derechos humanos. Tirant lo Blanch, Valencia, 2019, 270 pp., ISBN 978-84-1313-376-8.

\author{
http://dx.doi.org/10.18543/ed-68(2)-2020pp371-376
}

Para un estudioso de los derechos humanos se puede afirmar que encontrar una monografía dedicada a la dignidad humana es siempre motivo de interés y satisfacción, más si el estudio se dirige al análisis de concepto desde una perspectiva eminentemente práctica, cual es la garantía de los derechos fundamentales en relación con un grupo más vulnerable. Trabajos sobre la dignidad del trabajador, la dignidad de la mujer, también otros que versan sobre la dignidad del enfermo, del anciano o de la dignidad de las víctimas, son en buena parte tratados referidos a la dignidad humana en general, pero sobre todo interesan porque prueban además las dificultades, peligros y fallos del Estado constitucional cara a la materialización plena de los derechos fundamentales en determinados grupos y la forma en que se pueden solucionar dichos problemas de vigencia de los derechos iusfundamentales. Lo mismo puede decirse cuando se habla de la dignidad del embrión, sobre todo en su relación con la Bioética y los desarrollos médicos, la dignidad del niño y del adolescente. En este sentido, especialmente importante es la contribución de
Belda a la teoría y práctica de la dignidad humana en lo que se refiere a la protección jurídica, social y política de las personas con algún tipo de discapacidad. Se ha de tener en cuenta que este trabajo cobra un interés todavía mayor si tenemos también en cuenta el carácter de verdadero experto del autor en este tema, dado que dicha monografía es el resultado de una línea de investigación continuada desde que en 2012 publicara su libro titulado La protección constitucional $y$ legal de la lengua de signos ${ }^{1}$, primer estudio jurídico sobre esta materia en España. Además, ha publicado otros artículos igualmente imprescindibles para adentrarse en la discapacidad desde un punto de vista jurídico y desde su relación con el principio de no discriminación del art. $14 \mathrm{CE}$, como Derecho y discapacidad ${ }^{2}$ y Mujeres

${ }^{1}$ Enrique Belda Pérez-Pedrero, La protección constitucional y legal de la lengua de signos, Lex Nova, Valladolid, 2012.

${ }^{2}$ Enrique Belda Pérez-Pedrero, «Derecho y discapacidad», en Parlamento y Constitución, n. 19, Universidad de Castilla-La Mancha, 2018, pp. 309-319. 
con discapacidad ${ }^{3}$; y luego, también, ha dirigido un libro colectivo sobre el derecho a la educación de las personas con discapacidad que incluye trabajos de distintos autores sobre enseñanza y discapacidad desde una perspectiva de integración y mejora pedagógica, cara a eliminar fallos en el ámbito educativo y mejorar las posibilidades sociales y de futuro de los niños y jóvenes con algún tipo de discapacidad sensorial o física ${ }^{4}$.

El libro se abre con un interesante prólogo de Roberto Romboli, que introduce el tema como objeto de estudio propio del Derecho Constitucional, en particular por su conexión al concepto general de dignidad humana, los derechos fundamentales y, muy especialmente, con el principio de igualdad y no discriminación. En efecto, como ya apuntara Krüger, en el Estado «no existe razón que pueda justificar la exclusión de ninguna persona del status de ciudadano, independientemente de su capacidad absoluta, física o moral» ${ }^{5}$. Y es así que la

${ }^{3}$ Enrique Belda Pérez-Pedrero, «Mujeres con discapacidad: incidencia de la violencia de género y valoración desde el punto de vista de los derechos humanos y de la actuación del Estado Constitucional», en María Martín Sánchez (dir.), Estudio Integral de la Violencia de Género: un análisis teórico-práctico desde el Derecho y las Ciencias Sociales, Tirant lo Blanch, Valencia, 2018, pp. 115-135.

${ }^{4}$ Enrique Belda Pérez-Pedrero (dir.), Derecho a la educación de las personas con discapacidad, Tirant lo Blanch México, 2019.

${ }^{5}$ Herbert Krüger, Allgemeine Staatslehre, W. Kohlhammer, Stuttgart, 1966, p. 156. persona discapacitada, en cuanto un miembro más del cuerpo social, merece la misma atención en sus necesidades específicas que cualquier otro sujeto parte del colectivo; más dentro de un Estado que se califica a sí mismo como Estado de bienestar o Estado social. Por supuesto, el hecho de que la persona discapacitada posee exactamente igual dignidad con respecto a cualquier otro justifica la atención del Estado en sus necesidades especiales, pero antes también de forma jurídicopositiva por su propio status de ciudadano. De alguna forma, ambas razones justifican el refuerzo público cara a la materialización de los derechos fundamentales y sociales de estas personas. De este modo, para Romboli, la actuación activa del Estado en relación con la persona discapacitada parte del concepto de igualdad por su identificación como ciudadano común, pero a través de una interpretación específica de los derechos fundamentales que le corresponden. Y ello también, naturalmente, con independencia de que el individuo quede limitado en alguna de sus facultades sensoriales o físicas por nacimiento o, a posteriori, por enfermedad o algún accidente; hecho que también refuerza -sea dicho ya de paso- el esfuerzo del Estado en este campo, pues ninguno de nosotros queda exento, por vicisitudes de la vida, de tener que necesitar en algún momento o en el futuro atención especial.

Bajo el título «Las personas con discapacidad como sujetos de Derecho», se sitúa la parte introductoria del trabajo. Se trata de un estudio de situación del reconocimiento y garantía de los derechos del discapacitado 
en el tiempo. Belda reconoce ésta como una realidad relativamente reciente, dentro del constitucionalismo de mediados del Siglo XX, paralelo a la aparición real del Estado social y de bienestar. Las primeras acciones de protección se asentaban más bien, dice, en el marco caritativo-asistencial. Esta primera forma de acción, no carente de hitos importantes, como, por ejemplo, la creación de la ONCE (Organización Nacional de Ciegos Españoles) de 1938, inicia de alguna forma el desarrollo de reconocimiento de los derechos de las personas con discapacidad cada vez a mayor nivel, hasta la actual comprensión derivada de la comprensión iusconstitucional de las personas con discapacidad como «minoría», y de las medidas en pro de su integración total como discriminación positiva en conexión con el concepto de igualdad formal y material de los arts. 9.2 y 14 de la CE y, además, como derivación de acuerdos internacionales suscritos por España como, por ejemplo, La Convención Internacional de Nueva York sobre los Derechos de las Personas con Discapacidad de 13 de diciembre de 2006 (Instrumento de ratificación de 23 de noviembre de 2007). El autor explica el carácter vinculante en todo caso de la obligación estatal de promoción y materialización de los derechos de las personas con discapacidad, no sólo sobre la base del concepto de dignidad humana en general, sino también sobre la base ineludible de la misma forma en la que se configura el Estado social, es decir, de su compromiso de «asegurar a todos una digna calidad de vida» a través de la siempre obligatoria promoción a mayores niveles de eficacia de los derechos fundamentales y los derechos sociales; y luego, muy particularmente, sobre la base de la positivación constitucional del principio de igualdad material del art. 9.2 CE. Y téngase en cuenta que esta obligación del Estado se traspone también en una tarea mediata, ya que los poderes públicos deben contribuir progresivamente, ad calendas graecas, a la satisfacción evolutiva de las necesidades especiales de este colectivo, de la misma manera que en el caso de las necesidades humanas de cualquier ciudadano ${ }^{6}$. Belda indica que este mandato no refiere además por tanto un cumplimiento de mínimos, sino de materialización de derechos fundamentales en pleno contenido y fin, de la misma manera que en relación a cualquier otro ciudadano.

El Capítulo siguiente, dedicado a la «Dignidad para el Derecho Constitucional», es de una extensión mayor y analiza los fundamentos del concepto de dignidad de la persona en general en el Derecho Público y Constitucional. Esto significa que aquí se analiza la noción de dignidad humana y su sentido de positivación constitucional, muy particularmente desde su reconocimiento en el art. 10.1 CE, así como su interpretación jurídico-práctica por la doctrina, sobre todo en España, Alemania y México. A ello le sigue una profundización en las normas de rango internacional, muy especialmente de la Declaración Universal de los Derechos Humanos de

${ }^{6}$ Alberto Oehling de los Reyes, La dignidad de la persona, Dykinson, Madrid, 2010, pp. 465 y 466. 
1948 y otros instrumentos regionales conexos como el Convenio Europeo de Derechos Humanos de 1950 y la Convención Americana sobre Derechos Humanos de 1969; luego también del proceso de recepción en la Unión Europea. La parte del Capítulo dedicada a la Convención Internacional de Nueva York sobre los Derechos de las Personas con Discapacidad de 2006, como texto práctico de aplicación concreta del concepto de dignidad general en el caso de las personas discapacitadas, es también sobresaliente en su desarrollo metodológico y explicativo, también su análisis de los textos legales a través de los cuales se han traspuesto sus propuestas al Derecho interno, concretamente la Ley 26/2011, de 1 de agosto, de adaptación normativa a la Convención Internacional sobre los Derechos de las Personas con Discapacidad y el Real Decreto Legislativo $1 / 2013$, de 29 de noviembre, por el que se aprueba el Texto Refundido de la Ley General de derechos de las personas con discapacidad y de su inclusión social. Luego el Capítulo concluye con un examen exhaustivo del estado de situación de la noción en la jurisprudencia del Tribunal Constitucional, estudiando la función judicial-constitucional como un instrumento práctico de especial relevancia a efectos de dotar de plasticidad el concepto de dignidad de la persona del art. 10.1 CE en casos concretos en las relaciones públicas y privadas.

El Capítulo tercero es el que más profundiza en el objeto de investigación propuesto de forma independiente («Dignidad y discapacidad»). La pregunta inicial es si las personas con alguna discapacidad tienen un tipo distinto de dignidad. Por supuesto, la persona con discapacidad goza exactamente de la misma dignidad que cualquier otro ciudadano, pues, como ya dice de forma muy expresiva el Tribunal Constitucional alemán, «todos poseen dignidad sin consideración a sus características, sus capacidades, y su estatus social y sin que se pueda tener en cuenta su situación corporal o mental» (BVerfGE 87, 209, 228); a lo que se refiere Belda con dicha pregunta es a la determinación de resultados de la comprensión de la igual dignidad desde la perspectiva de la generalidad y no tanto desde la especialidad. Entiende el autor que la materialización de los derechos de la persona con discapacidad desde una perspectiva de integración e inclusión opera no desde su situación particular, sino desde su carácter como sujetos y ciudadanos de pleno derecho del Estado constitucional (sui juris), de lo cual deriva, en efecto, un derecho genérico a disfrutar de los beneficios del Estado social y de bienestar en una parte alícuota igual al resto de conciudadanos, con las particularidades que ello pueda suponer para evitar situaciones de segregación, en particular por vías de discriminación positiva, como ocurre con otros colectivos. De ahí, como también señala el autor, que la persona con discapacidad pueda disfrutar, según los casos, de una consideración preferente del Estado a efectos de refuerzo para materializar su derecho a la igualdad, a saber, de un comportamiento activo de éste que intente situar en la misma senda de desarrollo personal a estas personas como el del resto de la población, sin que, además, 
se produzcan fallos por discriminación.

Dentro del epígrafe titulado «La aparición de la dignidad en las relaciones jurídicas e institucionales de las personas con discapacidad», el autor introduce una interesante relación de casos prácticos de materialización de la noción de dignidad general en el caso concreto de los discapacitados. El primer ejemplo versa sobre el derecho al voto de la persona con discapacidad. Naturalmente, en tanto miembros del colectivo social, iguales en dignidad, también las personas discapacitadas deben de tener derecho a participar en el proceso electivo, pues lógicamente el asunto también va con ellos e, independientemente de cualquier factor, tienen su propia ideología y los mismos deseos que cualquier otro a optar por un programa electoral acorde a sus intereses ${ }^{7}$. En cambio, tradicionalmente, la Ley Orgánica 5/1985, de 19 de junio, del Régimen Electoral General, en su art. 3.1, posibilitaba en situaciones de discapacidad intelectual, deterioro cognitivo o enfermedad psíquica, la suspensión de su derecho de sufragio por vía de sentencia judicial firme que determinase expresamente la incapacidad para el ejercicio de dicho derecho fundamental. Hoy esta posibilidad ha sido eliminada por la Ley Orgánica 2/2018, de 5 de diciembre, reconociéndose el ejercicio del derecho participación política a la persona discapacitada sin ningún tipo de cortapisa y en régimen de igualdad al resto de ciudadanos.

${ }^{7}$ Alberto Oehling de los Reyes, La dignidad de la persona, cit., p. 413.
Belda analiza de forma convincente aquella incoherencia, sobre todo en conexión con la libertad ideológica (art. 16.1 CE) y el derecho a participar en asuntos políticos (art. 23.1), su pervivencia en el tiempo y evolución; incluyendo, además, un comentario sobre el Auto del Tribunal Constitucional 196/2016, de 28 de noviembre, que todavía validaba la facultad judicial de privación del derecho a las personas discapacitadas ${ }^{8}$. El segundo ejemplo se refiere a la materialización del derecho a la educación del art. 27 CE. Aquí Belda hace un análisis reflexivo de la necesidad de implementación a mayor grado de este derecho en el caso de personas con discapacidad como condición para favorecer la igualdad de oportunidades; de cómo se materializa el derecho a la formación de los discapacitados desde una perspectiva inclusiva, tanto en el plano de contenido de la educación, adaptación de currículos educativos, evaluación adecuada y dotación de instalaciones adaptadas para aquellos casos en que el derecho a la educación se pueda materializar mejor de ese modo. El tercer conjunto de ejemplos se refiere a la dignidad humana y el derecho a la igualdad desde el punto de vista del derecho a la vida, la integridad física, la libertad sexual y reproductiva, en cuya explicación se incluyen problemas derivados de las operaciones reproductivas

${ }^{8}$ Véase, sobre ello, Alberto Anguita Susi, «Realidad y perspectiva del derecho de sufragio activo de las personas con discapacidad intelectual», en Teoría y realidad constitucional, n. ${ }^{\circ}$ 44, UNED, Madrid, 2019, p. 428. 
relacionadas con la discapacidad, esterilizaciones involuntarias y abortos eugenésicos en razón a la discapacidad del nascituri, como también sobre la posibilidad de selección embrionaria para disminuir las posibilidades de nacimiento de personas con discapacidades congénitas. La relación de ejemplos termina con una breve explicación de otros problemas como, entre otros, eutanasia en relación con la discapacidad, la capacidad de obrar del discapacitado, la discriminación laboral, mujer y discapacidad, etc.

En definitiva, en esta monografía se realiza un estudio teórico-práctico sobre la protección social, política y jurídica de las personas con algún tipo de discapacidad, un análisis profundo que da respuesta a las exigencias de resolución de los posibles fallos del sistema en orden a la materialización plena de los derechos fundamentales y sociales de los discapacitados; la dignidad de la persona con discapacidad en el marco de la Constitución de 1978 y la Unión Europea, el concepto de «dignidad inherente» de las personas con discapacidad de la Convención Internacional de Nueva York, de 13 de diciembre de 2006, y el mandato de garantía de sus derechos en condiciones de igualdad y no discriminación. En cada uno de los temas tratados, Belda analiza con detalle su evolución y estado de situación actual, avances y retrocesos, su regulación vigente, su lógica jurídicoteórica, así como casos y ejemplos de carácter práctico. El estudio además no se limita al Derecho español, sino que se realiza con un pie en la doctrina extranjera, en particular alemana; luego, lo refiere también en conexión con el Derecho de la Unión Europea y el Derecho Internacional, que analiza con rigor y solvencia. La solidez de la formación técnica y científica del autor en este campo le posibilita trabajar esta complicada faceta de los derechos fundamentales y los derechos humanos no tanto desde la Filosofía, sino desde el plano del Derecho Constitucional positivo y con una intención de desarrollar una investigación eminentemente práctica. Por eso, la intención Enrique Belda de realizar este estudio sobre la dignidad humana en su relación con las personas con algún tipo de discapacidad, puesto al día, muy amplio y detallado, me parece digno de elogio, cuyo resultado final no sólo deja clara la calidad humana del autor, sino que vuelve a probar el nivel académico, científico y docente de este jurista.

Alberto Oehling de los Reyes

Profesor contratado doctor Universidad de las Islas Baleares (en excedencia) Universidad Complutense de Madrid 


\title{
Belda, Enrique, Dignidad y discapacidad. Una perspectiva desde los derechos humanos. Tirant lo Blanch, Valencia, 2019, 270 pp., ISBN 978-84-1313-376-8.
}

\author{
http://dx.doi.org/10.18543/ed-68(2)-2020pp371-376
}

\section{Copyright}

Estudios de Deusto es una revista de acceso abierto, lo que significa que es de libre acceso en su integridad. Se permite su lectura, la búsqueda, descarga, distribución y reutilización legal en cualquier tipo de soporte sólo para fines no comerciales, sin la previa autorización del editor o el autor, siempre que la obra original sea debidamente citada y cualquier cambio en el original esté claramente indicado

Estudios de Deusto is an Open Access journal which means that it is free for full access, reading, search, download, distribution, and lawful reuse in any medium only for non-commercial purposes, without prior permission from the Publisher or the author; provided the original work is properly cited and any changes to the original are clearly indicated. 